\title{
The value of intraoperative neurophysiological monitoring in tethered cord surgery
}

\author{
Eelco W. Hoving • Esther Haitsma • \\ Charlotte M. C. Oude Ophuis • Henricus L. Journée
}

Received: 8 April 2011 / Accepted: 15 April 2011 / Published online: 3 May 2011

(C) The Author(s) 2011. This article is published with open access at Springerlink.com

\begin{abstract}
Purpose The value of intraoperative neurophysiological monitoring (IONM) with surgical detethering in dysraphic patients has been questioned. A retrospective analysis of our series of 65 patients is presented with special focus on technical set-up and outcome.

Methods All patients were diagnosed with a tethered cord (TC) due to spinal dysraphism. A high-risk group (HRG) was determined consisting of 40 patients with a lipomyelomeningocele and/or a split cord malformation sometimes in combination with a tight filum terminale. The surgical procedure was a detethering operation in all cases performed by a single surgeon during a 9-year period (1999-2008). A standard set-up of IONM was used in all patients consisting of motor-evoked potentials (MEP) evoked by transcranial electrical stimulation (TES) and electrical nerve root stimulation. In young patients, conditioning stimulation was applied in order to improve absent or weak MEPs.

Results IONM responses could be obtained in all patients. Postoperative deterioration of symptoms was found in two patients of whom one patient belonged to the HRG. Mean maximal follow-up of all 65 patients was 4.6 years (median 4.1 years). Long-term deterioration of symptoms was found in 6 of 65 patients with a mean follow-up of 5 years (median 5.3 years).

Conclusion The use of IONM is feasible in all TC patients. The identification of functional nervous structures and continuous guarding of the integrity of sacral motor roots by IONM may contribute to the safety of surgical detethering.
\end{abstract}

E. W. Hoving $(\varangle) \cdot$ E. Haitsma • C. M. C. Oude Ophuis

H. L. Journée

Department of Neurosurgery,

University Medical Centre Groningen,

PO Box 30.001, 9700RB Groningen, The Netherlands

e-mail: e.w.hoving@nchir.umcg.nl
Keywords Spinal dysraphism · Tethered cord . Intraoperative neuromonitoring $\cdot$ Dethetering

\section{Introduction}

The morphology of spinal dysraphism is associated with tethered cord, including a wide variety of malformations like myelomeningocele, lipomyelomeningocele, tight filum terminale, split cord malformation, and dermoid sinus. Combinations of these anomalies are common. Patients with a tethered cord (TC) may develop symptoms during their life. Once symptoms have developed, the patient has a tethered cord syndrome (TCS) $[1,2]$. These symptoms may either be neurological, urological, orthopedic, or pain. In adult TCS patients, pain is often the most predominant symptom [3]. Cutaneous stigmata indicate the presence of spinal dysraphism. Hence, asymptomatic TC patients can be diagnosed. The natural history of TC in spinal dysraphism is partly understood. It remains difficult to discriminate between the development of symptoms due to TCS or to myelodysplasia in these patients. In 53 asymptomatic TC patients with a spinal lipoma, Kulkarni et al. found that $33 \%$ of them became symptomatic during a 9-year period of follow-up [4]. Prophylactic detethering in a comparable category of lipoma patients could not prevent the development of symptoms in $50 \%$ of patients after a 5 -year follow-up [5].

The goal of surgery is to detether the cord by disconnecting its aberrant tightening attachments, and to relieve the cord from continuous stretching forces. Tethering structures may consist of lipoma, fibrous bands, non-functional nervous tissue, or a dermoid sinus tract [5]. Peroperatively it can be difficult to distinguish between functional neural tissue and these non-functional tethering structures. Most postoperative complications are reversible problems like cerebrospinal 
fluid leaks or wound infections. Irreversible neurological or urological deterioration may occur in a minority of cases. Patients with lipomyelomeningocele (LMC) or split cord malformation (SCM) harbor most of these postoperative irreversible changes and consequently these two categories were defined as high-risk group (HRG).

The use of intraoperative neurophysiological monitoring (IONM) may contribute to the safety in TC surgery $[1,6-10]$. We present a series of 65 patients with a wide variety of morphology of spinal dysraphism. All patients were operated for reasons of a tethered cord with the peroperative use of IONM. The feasibility of the use of IONM in different age groups and its potential contribution to the safety of the procedure are investigated.

\section{Material and methods}

\section{Subjects}

In this retrospective study, over a 9-year period (1999-2008), 65 subsequent patients received detethering surgery in the conus cauda region. All the operations were performed by a single surgeon (EWH).

The mean age of the patients was 22.0 years (median 11.5 years, range $0.1-72.7$ years). Sex distribution showed 24 males $(37 \%)$ and 41 females. All patients were diagnosed with a tethered cord due to spinal dysraphism. A HRG was identified for patients with LMC and/or a $\mathrm{SCM}$, sometimes in combination with a tight filum terminale (TFT). The HRG contained 40 patients (Table 1). Of the remaining patients, two were diagnosed with a myelomeningocele (MMC), ten with a TFT, five with a dermoid sinus, and three patients had a combination of a TFT and a filum lipoma. Another five patients had been diagnosed with other combinations (two TFT + syringomyelia, one MMC + syringomyelia, one MMC + TFT, one dermoid + TFT).

Table 1 Morphology tethered cord

\begin{tabular}{lcc}
\hline & $n$ & $\%$ \\
\hline Number of patients ( $n, \%$ male) & 65 & 37 \\
Age (mean 22.0 years, SD 22.4 years) & - & - \\
Diagnoses ( $n$, \% of total) & & \\
- High-risk group: lipomyelomeningocele (LMC)/split cord & 40 & 62 \\
$\quad$ malformation (SCM) + tight filum terminale (TFT) & & \\
- Tight filum terminale (TFT) & 10 & 15 \\
- Myelomeningocele (MMC) & 2 & 3.1 \\
- Dermoid sinus & 5 & 7.7 \\
- Tight filum terminale (TFT) + filum lipoma & 3 & 4.6 \\
- Other & 5 & 7.7 \\
\hline
\end{tabular}

Of all patients, $77 \%$ were symptomatic with a TCS $(n=50)$. Neurological deficits were present in 32 patients $(64 \%$ of symptomatic patients) (Table 2). Urological deficits were present in 27 patients (54\% of symptomatic patients). A group of 14 patients presented with orthopedic deficits, consisting of scoliosis, and 28 patients experienced pain ( $28 \%$ of symptomatic patients and $56 \%$ of symptomatic patients respectively). A combination of these symptoms was present in 34 patients ( $70 \%$ of all symptomatic patients).

Methods

For intraoperative neurophysiological assessment, two modalities were applied on all patients. The modalities used were transcranial electrical stimulation and direct root stimulation. Both consist of recorded evoked muscular motor potentials (mMEP) that were evoked by either transcranial electrical stimulation (TES) or by direct stimulation of nerve roots in the surgical exposed area. The bulbocavernosus reflex (BCR), a third IONM modality to monitor the sacral sensory roots and neural circuitry, was later added to the protocol but was not considered in this study. Direct spinal stimulation has been used to exclude motor function of the surgically identified filum terminale or other tethered structures, whereas TES-mMEP was recorded for monitoring the integrity of sacral motor roots and neural circuitry in the lower spinal cord.

\section{IONM procedure}

TES was performed following a standard procedure applying a custom made voltage stimulator (NeuroGuard@); JS Center, Bedum, The Netherlands). The stimulator output impedance was $22 \Omega$ and had a current limit of 1 A. Two needle electrodes were placed on the head subcutaneously at the anatomical location C3 and C4 (EEG 10-20 system). Surface electrodes $\left(3 \mathrm{M}^{\circledR}\right.$ ECG) were used to measure mMEP on three muscle pairs (Fig. 1). These three muscle pairs were the quadriceps muscles (L2-4), anterior tibial muscles (L4-5), and gastrocnemius muscles (S1-2). MEP responses were also measured in the external anal sphincters by using needle electrodes (S2-4).

Table 2 Tethered cord syndrome

\begin{tabular}{lll}
\hline Symptomatic patients $(n=50)$ & Sole symptom, $n$ & Total, $n^{\text {a }}$ \\
\hline Neurological deficits & 2 & 32 \\
Urological deficits & 4 & 27 \\
Orthopedic deficits & 7 & 14 \\
Pain & 3 & 28 \\
\hline
\end{tabular}

a 34 patients had multiple symptoms 
Fig. 1 Transcranial electrical stimulation and direct root stimulation. Schematic display of used IONM techniques. Muscle motorevoked potentials were obtained from the following muscle pairs: external anal sphincter, $\mathrm{m}$. quadriceps femoris, $m$. tibialis anterior and $\mathrm{m}$. gastrocnemius. a Transcranial electrical stimulation at anatomical location $\mathrm{C} 3$ and $\mathrm{C} 4$. Responses in the form of mMEPs were recorded as at the lower extremities and external anal sphincter. b Direct nerve root stimulation was performed intraoperatively by the surgeon with a monopolar and a bipolar probe. Responses were recorded as with TES-MEP
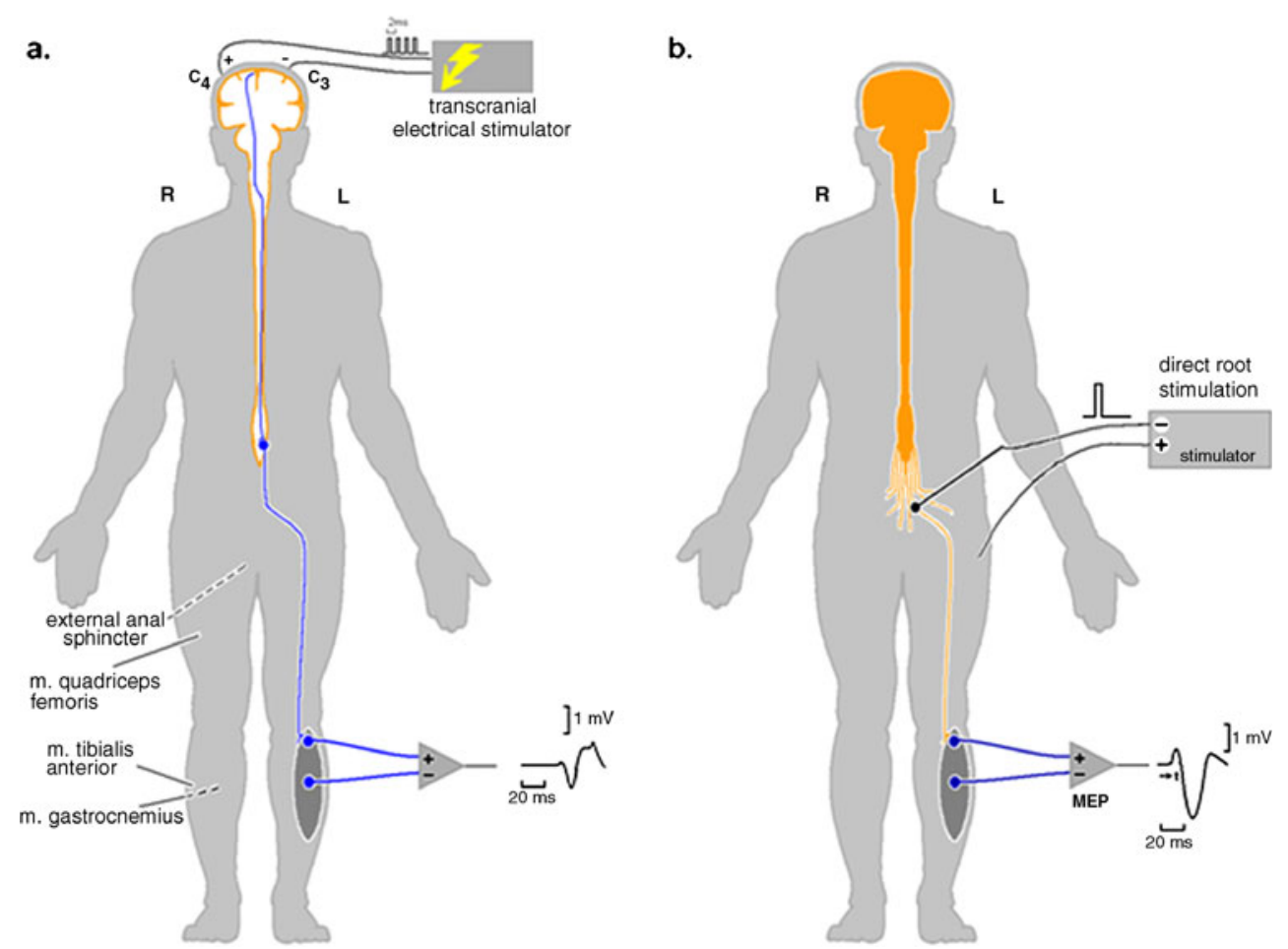

In a standard protocol, the TES stimulus consisted of a train of four and later on five pulses. The interpulse interval (IPI) was optimized for maximum MEP amplitudes in a range of $0.8-2 \mathrm{~ms}$. The pulse width was $100 \mu \mathrm{s}$. TES pulses were administered either monophasic with alternating polarity or biphasic. To determine the optimal stimulation voltage, an intensity curve was made at predefined increments of the TES voltage starting at $0 \mathrm{~V}$. Voltage thresholds $\left(V_{\mathrm{th}}\right)$ were defined as the start of a distinct increase of the MEP amplitude.

The criterion level for the presence of a MEP response originating from the CNS was the presence of an MEP amplitude well above $100 \mu \mathrm{V}$. Subsequent optimizing procedures were performed until all MEP amplitudes from the leg muscles were above this criterion.

The optimizing procedures consisted of measurement of MEP amplitudes at a stimulation voltage of $20-50 \%$ above $V_{\text {th }}$, function of IPI, and conditioning stimulation. Increasing the number of pulses and use of double train stimulation (DTS) were the conditioning techniques. DTS consisted of applying two trains of pulses with a short inter train interval (ITI) $[11,12]$. Optimal parameters for IPI, $n$, and ITI were selected for use during surgery. A voltage curve at the optimal parameter settings was performed preoperatively.

TES-MEP recordings were reassessed prior to and during the course of surgical intervention for detection of functional neural effects. Direct nerve root stimulation for identification and mapping of vital nervous tissue in the operated area was performed by the surgeon. A monopolar and/or a bipolar probe were used as shown in Fig. 2 applying $200 \mu$ s voltage pulses. When the voltage threshold was over three times the voltage threshold of a prior stimulated nerve root in the operating field, the structure was resected.

Subsequently a TES-MEP stimulus was applied in order to reassess the responses of the vital neural tissue after detethering. The procedure ended with final transcranial measurements.

\section{Anesthetics}

No muscle relaxants were given during surgery because of the negative effect on muscle responses. Short working muscular-blocking agents were only given during intubation. All patients received opiates for sedation (sufentanil or remifentanil). The first eight to ten patients of this series received sevoflurane as halogenated agent. As this inter-

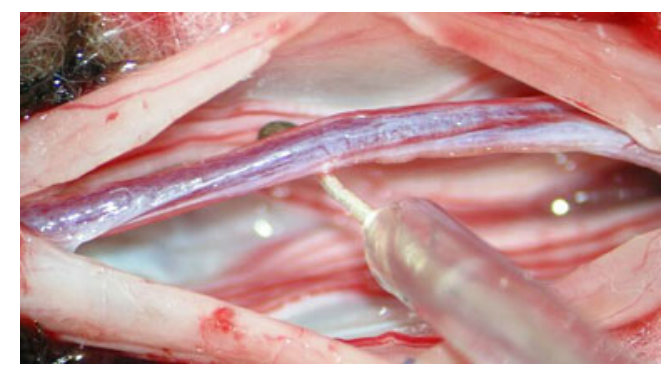

Fig. 2 Direct stimulation of a terminal filum with a monopolar probe. During surgery, direct spinal stimulation was performed in order to objectively discriminate between nerve roots and non-nervous tissue like the terminal filum. With a threshold of at least three times the threshold of an identified nerve root, non-nervous tissue was dissected 
fered with the monitoring procedure, the anesthetic protocol was changed to total intravenous anesthesia (TIVA) with propofol $2 \%$. Three patients received sevoflurane only during induction, after which TIVA was started. For reason of constant amplitude MEP readings, propofol was maintained at a constant level whereas the sedation level was controlled by adaptation of the opioid level. Bolus application was avoided.

\section{Scoring}

Scoring of the overall function of all patients was performed based on medical record. All patients were scored before and directly after surgery. The overall function was also determined at maximal follow-up. Four scoring parameters were identified: neurological, urological, and orthopedic symptoms as well as pain. The scoring method consisted of scoring values from $0-3$, ranging from asymptomatic (0), minimal/mild (1), moderate (2) to severe (3) symptoms (Table 3). Neurological deficits were determined by physical examination, using the strength scale of the medical research council. Urological deficits were determined by the results from video urodynamics (VU). Orthopedic deficits, consisting only of scoliosis in this series, were determined by the extent of scoliosis based on a progression over time of the Cobb angle with more than $5^{\circ}$. Also a pain questionnaire was used.

\section{Results}

The safety of the surgical procedure has been determined by the differences in pre- and postoperative scoring. Feasibility was determined by obtaining reliable measurements.

\section{Feasibility}

At the start of this study, 11 patients were sedated with halogenated agents (sevoflurane). This had a profound depressing effect on TES-MEP responses. In six out of eight patients receiving halogenated agents as sole sedative, it was impossible to obtain responses. In three patients receiving halogenated agents for induction, TESMEP was feasible after switch to TIVA. Hence the anesthetic regime was changed to TIVA. Direct root stimulation was possible in all patients and reliable measurements could be obtained.

Safety

The pre- and postoperative scores of three scoring parameters were presented (Table 3). Postoperative changes in orthopedic scores were not expected. Neurologically four out of 65 patients improved, 60 patients remained stable (Table 4). Urological score showed one patient with improvement and 63 patients remaining stable. Both urological and neurological deterioration occurred only in one MMC patient. Pain score showed that 20 patients improved, 44 patients remained stable, and one patient deteriorated.

Neurological outcome showed three patients with a single step improvement in scoring and one patient with a two step improvement (Table 5). Only one patient showed a single step deterioration in scoring. The urological score showed one patient with an improvement of one step. Another patient showed a deterioration of three steps. Improved pain score by a single step occurred in 14 patients, in five patients by two steps, and in one patient even by three steps. These patients were predominantly adults (mean age 34.5 years, range 1-72 years). A single patient in pain experienced an increase in pain scoring by one step.

\section{High-risk group}

The HRG was considered separately. A single step improvement in neurological scoring occurred in two patients and one patient showed a two step improvement in neurological scoring (Table 6). The majority of 37 patients remained neurologically stable. Urological outcome was stable in all patients. A decrease in pain score was seen in 12 patients. In two of these patients, there was an improvement in scoring with two steps and in the remaining ten there was an improvement in scoring with one step. One patient showed a single step increase in pain score. In the HRG, there was no neurological or urological deterioration.

\section{Non-high-risk group}

The remaining non-high-risk group (nHRG) showed improvement of neurological outcome by one step in one

Table 3 Scoring of deficits

\begin{tabular}{lllll}
\hline Deficit & Neurological & Urological & Orthopedic & Pain \\
\hline $0=$ none & - & - & - & - \\
$1=$ minimal & Sensibility & VU abnormal & Scoliosis minimal & Pain minimal \\
$2=$ moderate & Motor minimal $(>4)$ & Bladder residue & Scoliosis moderate & Pain moderate \\
$3=$ severe & Motor paresis $(<4)$ & Incontinence & Scoliosis severe & Pain severe \\
\hline
\end{tabular}


Table 4 Surgical outcome

\begin{tabular}{llll}
\hline Deficits & Improved & Stable & Deteriorated \\
\hline Neurological & 4 & 60 & $1^{\mathrm{a}}$ \\
Urological & 1 & 63 & $1^{\mathrm{a}}$ \\
Pain & 20 & 44 & 1 \\
\hline
\end{tabular}

${ }^{\mathrm{a}}$ This deterioration in scoring occurred in one patient

patient (Table 6). In a single patient, neurological deterioration occurred with one step. Urological scoring in these patients showed a three step deterioration in one patient, while in another patient, a single step improvement was found. In total eight patients showed a decrease in pain. The scoring showed four patients with one step improvement, three patients with two steps, and one patient with three steps.

\section{Prophylactic patients}

Fifteen asymptomatic patients received prophylactic detethering surgery. The mean age of these asymptomatic patients was 5.1 years (SD 8.9 years, range 32.4 years, median 1.2 years), while TCS patients in our series had a mean age of 27.1 years $(n, 50$ : SD, 22.8 years; median, 20.3 years). A group of seven prophylactic patients belonged to the HRG.

Follow-up

The mean follow-up of all 65 patients was 4.6 years (SD 2.5 years, median, 4.1 years, range 9.2 years, min-max 1.1-10.3 years). Scoring of deficits directly after surgery and at maximum follow-up has been compared. Neurological outcome showed that 60 patients remained stable and one patient deteriorated (Table 7). The scoring of four patients improved. Urological outcome showed that 57 patients remained stable, two patients deteriorated, and six patients improved. All patients with deterioration of

Table 5 Absolute scoring surgical outcome $(n=65)$

\begin{tabular}{lllll}
\hline & Score difference & Neurologic, $n$ & Urologic, $n$ & Pain, $N$ \\
\hline Improved & +1 & 3 & 1 & 14 \\
& +2 & 1 & - & 5 \\
& +3 & - & - & 1 \\
Subtotal & & 4 & 1 & 20 \\
Stable & & 60 & 63 & 44 \\
Deteriorated & -1 & 1 & - & 1 \\
& -2 & - & - & - \\
& -3 & - & 1 & - \\
Subtotal & & 1 & 1 & 1 \\
\hline
\end{tabular}

Table 6 Surgical morbidity high-risk group versus non-high-risk group

\begin{tabular}{|c|c|c|c|c|}
\hline \multirow[b]{2}{*}{ Scoring deficits } & \multicolumn{2}{|c|}{ HRG $(n=40)$} & \multicolumn{2}{|c|}{ nHRG $(n=25)$} \\
\hline & $n$ & $\%$ & $n$ & $\%$ \\
\hline \multicolumn{5}{|l|}{ Neurological } \\
\hline Improved & 3 & 7.5 & 1 & 4.0 \\
\hline Stable & 37 & 93 & 23 & 92 \\
\hline Deteriorated & 0 & 0.0 & 1 & 4.0 \\
\hline \multicolumn{5}{|l|}{ Urological } \\
\hline Improved & 0 & 0.0 & 1 & 4.0 \\
\hline Stable & 40 & 100 & 23 & 92 \\
\hline Deteriorated & 0 & 0.0 & 1 & 4.0 \\
\hline \multicolumn{5}{|l|}{ Pain } \\
\hline Improved & 12 & 30 & 8 & 32 \\
\hline Stable & 27 & 68 & 17 & 68 \\
\hline Deteriorated & 1 & 2.5 & 0 & 0.0 \\
\hline
\end{tabular}

$H R G$ high-risk group, $n H R G$ non-high-risk group

urological function were below 10 years of age. Orthopedic outcome showed that three patients deteriorated and one patient improved. The other 61 patients remained stable. The pain score remained stable in 63 patients, and the score improved in two patients. The scoring difference per patient is shown in Table 7. All symptomatic patients showed a general deterioration in a scoring of $8 \%$ at maximal followup $(n=50)$. General deterioration at maximal follow-up occurred only in patients aged younger than 18 years.

\section{Follow-up HRG}

The 40 patients of our HRG had a mean follow-up of 5.2 years (SD 2.5 years, median 5.0 years, range 9.2 yrs). Improved neurological scoring was found in three patients, two improved by a single step, and one by two steps (Table 8). The majority of 37 patients remained stable. Neurological outcome showed no patients with deterioration of scoring. Improvement of urologic scoring occurred in four patients: two patients improved with one step in scoring, one patient with two steps, and another patient with three steps in scoring. Stable urology at maximal follow-up was seen in 35 patients. Urologic outcome showed one patient who deteriorated with three steps in scoring. Improvement of orthopedic outcome occurred in one patient with one step in scoring, and 36 patients remained stable. Orthopedic outcome showed three patients who deteriorated with one step in scoring. Pain relieve was seen in two patients, for one patient with one step, and for the other with three steps in scoring. The majority of 38 patients remained stable. There was no deterioration in pain outcome. 
Table 7 Follow-up outcome $(n=65)$

\begin{tabular}{llllll}
\hline & Score difference & $\begin{array}{l}\text { Neurological, } \\
n(\%)\end{array}$ & $\begin{array}{l}\text { Urological, } \\
n(\%)\end{array}$ & $\begin{array}{l}\text { Orthopedic, } \\
n(\%)\end{array}$ & $\begin{array}{l}\text { Pain, } \\
N(\%)\end{array}$ \\
\hline Improved & +1 & 3 & 4 & 1 & 1 \\
& +2 & 1 & 1 & - & - \\
& +3 & - & 1 & - & 1 \\
Subtotal & & $4(6.2)$ & $6(9.2)$ & $1(1.5)$ & $2(3.1)$ \\
Stable & & $60(92)$ & $57(88)$ & $61(94)$ & $63(97)$ \\
Deteriorated & -1 & 1 & 1 & 3 & - \\
& -2 & - & - & - & - \\
Subtotal & -3 & - & 1 & - & - \\
\hline
\end{tabular}

\section{Follow-up $n H R G$}

The 25 patients who were not part of the HRG had a mean follow-up of 3.6 years (SD 2.2 years, median 2.9 years, range 4.8 years). A single patient showed improvement in neurological function with one step and 23 patients remained stable (Table 8). Neurological deterioration occurred in one patient with one step in scoring. Urological improvement occurred in two patients with one step in scoring. Stable urology scoring was seen in 22 patients and deterioration occurred in one patient with one step in scoring. Orthopedic and pain outcome were stable in all 25 patients.

Table 8 Follow-up outcome high-risk group versus non-high-risk group

\begin{tabular}{|c|c|c|c|c|}
\hline & \multicolumn{2}{|c|}{ HRG $(n=40)$} & \multicolumn{2}{|c|}{ nHRG $(n=25)$} \\
\hline & $n$ & $\%$ & $n$ & $\%$ \\
\hline \multicolumn{5}{|l|}{ Neurological } \\
\hline Improved & 3 & 7.5 & 1 & 4.0 \\
\hline Stable & 37 & 93 & 23 & 92 \\
\hline Deteriorated & 0 & 0.0 & 1 & 4.0 \\
\hline \multicolumn{5}{|l|}{ Urological } \\
\hline Improved & 4 & 10 & 2 & 8.0 \\
\hline Stable & 35 & 88 & 22 & 88 \\
\hline Deteriorated & 1 & 2.5 & 1 & 4.0 \\
\hline \multicolumn{5}{|l|}{ Orthopedic } \\
\hline Improved & 1 & 2.5 & 0 & 0.0 \\
\hline Stable & 36 & 90 & 25 & 100 \\
\hline Deteriorated & 3 & 7.5 & 0 & 0.0 \\
\hline \multicolumn{5}{|l|}{ Pain } \\
\hline Improved & 2 & 5.0 & 0 & 0.0 \\
\hline Stable & 38 & 95 & 25 & 100 \\
\hline Deteriorated & 0 & 0.0 & 0 & 0.0 \\
\hline
\end{tabular}

$H R G$ high-risk group, $n H R G$ non-high-risk group

\section{Follow-up prophylactic group}

The mean follow-up of the 15 asymptomatic patients was 4.4 years (SD 2.5 years, median 3.4 years, range 7.5 years). Of this prophylactic group, seven patients were also part of the HRG. Two of them showed deterioration. The first HRG patient suffered from urologic deterioration with three steps in scoring. The other HRG patient showed a single step deterioration in orthopedic scoring.

\section{Discussion}

Feasibility

IONM is a method which has been proven to be technically feasible even in younger age [11, 12]. Responses can always be obtained when no halogenated agents are administered and patients do not have severe neurological deficits [13, 14]. In our series, both transcranial electrical stimulation and direct electrical root/ nerve stimulation appear to be technically feasible, even in very young children. Technical adjustments like double-train stimulation may be required more often in young children. The presence of immature myelinated corticospinal fibers in this group of patients may desynchronize the transportation of action potentials on their way to the motor neurons. This often results in failure to elicit muscle MEPs. Conditioning by double-train stimulation compensates the relative hyperpolarisation of motorneurons resulting in stable MEP responses with high amplitudes $[15,16]$

The responses of the anal sphincter muscles are generated by the lower sacral nerves S2-5, and they represent the integrity of the bladder function as well. This type of monitoring of urological function in our series appears to be effective in maintaining bladder function in most patients, both postoperatively and during follow-up. Only one patient deteriorated with three steps to incontinence. This patient had a myelocystocele and was operated 
at the age of 1 month. This diagnosis is a separate entity in the spectrum of spinal dysraphism [17, 18]. During surgery, the nerve roots could easily be recognized along the wall of the sack, but its handling and repositioning into the spinal canal appeared to be hazardous. IONM was of limited value during surgical detethering since all nerve roots could be saved at least anatomically.

The continuous use of the BCR has not been used in a standard fashion at that time of surgery in this series. BCR might have had an additional value by indicating deterioration of functions of the sacral nerves in this particular young patient with myelocystocele. Currently BCR is also used in our standard IONM set-up of tethered cord surgery.

\section{Safety}

No direct postoperative neurological and/or urological deterioration was found in all 40 patients of the HRG while only one patient suffered from an increase in pain. The absence of neurological and urological deterioration in this technically challenging group illustrates a potential beneficiary effect of the use of IONM to the safety of the procedure [8]. In the $\mathrm{nHRG}$, only one patient deteriorated postoperatively in both neurologic and urologic function. As expected for the $\mathrm{nHRG}$ positive, results were found similar to the HRG $[11,19]$.

In our series, a technique of partial resection of the lipoma was used. Recently Pang et al. compared the postoperative neurological deterioration in a historical group with partial resection and a more recent total resection group with the use of IONM. The neuro-urologic complication rate appeared to be similar in both groups, $4.2 \%$ and $5.2 \%$, respectively [20, 21]. The postoperative results in our series are at least comparable to these figures.

Pain can effectively be treated as a symptom of TCS especially in adult patients [3]. The improvement of pain directly postoperatively in $36 \%$ of all symptomatic pain patients corroborates this in our series. Only one patient deteriorated progressively after surgery. This patient had a lipoma and was aged 60 years old. The patient had suffered from progressive neurological and urological deterioration before surgery. Peroperatively only a partial resection of the lipoma with a partial detethering could be accomplished. This partial detethering might have resulted in increased strain and tension in the caudal nerve roots with the subsequent increase in pain. In this case, the technique of total resection of the lipoma, as proposed by Pang, might have resulted in a better outcome.

\section{Follow-up}

Remarkably 20\% (13 patients) of the patients improved during follow-up. This included four patients with neurolog- ical improvement and six with urological improvement, both not only confirmed by questionnaire but also by physical neurological exam and video urodynamics. A majority of these patients belonged to the HRG (10/13). At maximum follow-up, six patients had deteriorated. Of these six patients, four belonged to the HRG. No neurological deterioration had occurred in the HRG. The overall deterioration was less than expected for the HRG. The absence of neurological deterioration may imply that no retethering has occurred and lasting effective detethering has been achieved in this technically challenging group. Although our FU is relatively short and our series is small, our results indicate the maintenance of neurological function. This promising outcome may be related to a beneficial contribution of IONM in spite of the partial resection of the lipoma. Maximal detethering may protect the patient for recurrent symptoms. The prophylactic group did not show any neurological deterioration during follow-up (seven HRG/eight nHRG). Pang et al. identified in his series the young asymptomatic patients to benefit most of radical resection. One should strive for a large cord sack ration in these patients. In our much smaller series, we focused on maximal detethering without maximal resection of the lipoma. This strategy appears also to be effective.

\section{Conclusions}

- IONM is technically feasible in all our patients. Concerning safety, there was a stable or improved neurological score in 64 out of 65 patients. No neurological or urological deterioration occurred in our HRG ( $n=40)$, while the follow-up of this group showed stable or improved neurology scores.

- Standard use of IONM should be considered in TCS surgery in order to improve safety.

- A potential positive effect of IONM on the efficacy of surgery might be present.

Open Access This article is distributed under the terms of the Creative Commons Attribution Noncommercial License which permits any noncommercial use, distribution, and reproduction in any medium, provided the original author(s) and source are credited.

\section{References}

1. Lew SM, Kothbauer KF (2007) Tethered cord syndrome: an updated review. Pediatr Neurosurg 43:236-248

2. Yamada S, Colohan AR, Won DJ (2009) Tethered cord syndrome. J Neurosurg Spine 10:79-80

3. Yamada S, Lonser RR (2000) Adult tethered cord syndrome. J Spinal Disord 13:319-323 
4. Kulkarni AV, Pierre-Kahn A, Zerah M (2004) Conservative management of asymptomatic spinal lipomas of the conus. Neurosurgery 54:868-873

5. Pierre-Kahn A, Zerah M, Renier D, Cinalli G, Sainte-Rose C, Lellouch-Tubiana A, Brunelle F, Le MM, Giudicelli Y, Pichon J, Kleinknecht B, Nataf F (1997) Congenital lumbosacral lipomas. Childs Nerv Syst 13:298-334

6. Paradiso G, Lee GY, Sarjeant R, Hoang L, Massicotte EM, Fehlings MG (2006) Multimodality intraoperative neurophysiologic monitoring findings during surgery for adult tethered cord syndrome: analysis of a series of 44 patients with long-term follow-up. Spine (Phila Pa 1976) 31:2095-2102

7. Quinones-Hinojosa A, Gadkary CA, Gulati M, von Koch CS, Lyon R, Weinstein PR, Yingling CD (2004) Neurophysiological monitoring for safe surgical tethered cord syndrome release in adults. Surg Neurol 62:127-133

8. Valentini LG, Visintini S, Mendola C, Casali C, Bono R, Scaioli W, Solero CL (2005) The role of intraoperative electromyographic monitoring in lumbosacral lipomas. Neurosurgery 56:315-323

9. Kothbauer KF, Deletis V (2010) Intraoperative neurophysiology of the conus medullaris and cauda equina. Childs Nerv Syst 26:247-253

10. Kothbauer KF, Novak K (2004) Intraoperative monitoring for tethered cord surgery: an update. Neurosurg Focus 16:E8

11. von Koch CS, Quinones-Hinojosa A, Gulati M, Lyon R, Peacock WJ, Yingling CD (2002) Clinical outcome in children undergoing tethered cord release utilizing intraoperative neurophysiological monitoring. Pediatr Neurosurg 37:81-86

12. Sala F, Krzan MJ, Deletis V (2002) Intraoperative neurophysiological monitoring in pediatric neurosurgery: why, when, how? Childs Nerv Syst 18:264-287
13. Wang AC, Than KD, Etame AB, La MF, Park P (2009) Impact of anesthesia on transcranial electric motor evoked potential monitoring during spine surgery: a review of the literature. Neurosurg Focus 27:E7

14. Langeloo DD, Lelivelt A, Louis JH, Slappendel R, de Kleuver M (2003) Transcranial electrical motor-evoked potential monitoring during surgery for spinal deformity: a study of 145 patients. Spine (Phila Pa 1976) 28:1043-1050

15. Journee HL, Polak HE, de Kleuver M, Langeloo DD, Postma AA (2004) Improved neuromonitoring during spinal surgery using double-train transcranial electrical stimulation. Med Biol Eng Comput 42:110-113

16. Journee HL, Polak HE, de Kleuver M (2007) Conditioning stimulation techniques for enhancement of transcranially elicited evoked motor responses. Neurophysiol Clin 37:423-430

17. Rossi A, Piatelli G, Gandolfo C, Pavanello M, Hoffmann C, Van Goethem JW, Cama A, Tortori-Donati P (2006) Spectrum of nonterminal myelocystoceles. Neurosurgery 58:509-515

18. Muthukumar N (2007) Terminal and nonterminal myelocystoceles. J Neurosurg 107:87-97

19. Pang D, Wilberger JE Jr (1982) Tethered cord syndrome in adults. J Neurosurg 57:32-47

20. Pang D, Zovickian J, Oviedo A (2010) Long-term outcome of total and near-total resection of spinal cord lipomas and radical reconstruction of the neural placode, part II: outcome analysis and preoperative profiling. Neurosurgery 66:253-272

21. Pang D, Zovickian J, Oviedo A (2009) Long-term outcome of total and near-total resection of spinal cord lipomas and radical reconstruction of the neural placode: part I-surgical technique. Neurosurgery 65:511-528 